Volume 2 No. 2, Agustus 2019

\title{
THE USE OF PRONUNCIATION BINGO GAME TO IMPROVE
}

\section{STUDENTS' PRONUNCIATION}

\author{
Ahmad Suhaili ${ }^{1}$, Asep Kurniawan ${ }^{2}$ \\ STIT Al-Khairiyah, STKIP Banten \\ Cilegon, Indonesia \\ ahmadsuhaili618@gmail.com \\ asepkurniawan010487@gmail.com
}

\begin{abstract}
This research is mainly purposed to find out whether there is influence of using pronunciation bingo game to improve students' pronunciation at first grade in MTs Alkhaeriyah Kepandean Ciruas Serang. The game is applied by experiment class and control class. The method of this research is true-experiment research. The population in this research is the first grade in MTs Alkhaeriyah Ciruas Serang. The writer took three classes with total number of the first grade are 132 Students. They are three classes, 44 Students from class VIIA and 44 Students from class VIIB and 44 Students from class VIIC. The writer took 60 students as sample and uses random sampling because the writer uses true-experiment method. To analyze this research the writer uses T-test method and to get the data the writer uses observation technique, and test as instrument of research. The observation is to know the students' pronunciation at the first grade in MTs Alkhaeriyah Keoandean Ciruas Serang, while, test is to know the influence use of pronunciation bingo game to improve students' pronunciation at the first grade in MTs Alkheriyah Kepandean Ciruas Serang. After having analysis data, there is influence using of pronunciation bingo game to improve students' pronunciation between experiment class and control class, based on the calculation is know that $\mathbf{t}_{\text {table }}$ with level significance $5 \%=2,00$ and with level significance $1 \%=2,65$ so to $\mathrm{o}_{\text {bservation }} \mathrm{n}=20,34>\mathbf{t}_{\text {table }}=2,00$ or to $\mathrm{o}_{\text {bservation }} \mathrm{n}=20,34>\mathbf{t}_{\text {table }}=2,65$.the conclusion is that the writer reject $\mathrm{H}_{0}: \mathrm{t}_{\mathrm{o}}<\mathrm{t}_{\mathrm{t}}$. It means there is no influence the use of pronunciation bingo game to improve students' pronunciation at the first grade in MTs Alkhaeriyah Kepandean Serang and receive Ha : $t_{0}>t_{t}$. it means there is influence the use of pronunciation bingo game to improve students' pronunciation at the first grade in MTs Alkhaeriyah Kepandean Ciruas Serang.
\end{abstract}

Keyword: Pronunciation, Bingo Game, experiment research, Control Class, Al-khairiyah 


\section{INTRODUCTION}

Communication can be trouble when the listener and speaker are from different linguistic backgrounds.. Even student with significant education experience can have problems in pronunciation. Perhaps early pronunciation was taught by non-native speaker who his self has oral production problems.

The pronunciation is one of component of English language especially in developing speaking skill. Pronunciation is important if no one understands what a speaker says, it does not matter how many words or tenses he knows so he can communicate. Students has different problem according to their mother tongues. Then, teaching pronunciation is to avoid the mispronunciation between speakers in their communication. The interfering teaching English pronunciation to the second or foreign language not only in terms of accent it mean intonation some of country has different accent, feature of Java accents may lead to Java people sounding. The second can be interfered tone or mood of utterance.

A lot of pronunciation teaching tends to be done in response to errors which students make in the classroom (Kelly, 2000). It means that teaching pronunciation is not prepared before.
According to Kenworthy Joanne, et.al (2002:97) says that "The English spelling system is rich in both regularities and irregularities which present problems to non-native learners (and to Englishspeaking children learning to write their language)".

Teaching pronunciation is not easy to do, many teachers get difficulty in teaching pronunciation to their students. Then, this study will present a pronunciation teaching strategy to intermediate school students. Because of the problem mention above the writer wrote about "The Use of Pronunciation bingo Game to improve students' pronunciation.

Pronunciation bingo is one of games that used by teachers in teaching pronunciation. It is a really popular pronunciation game and it seems a little complicated at first but it's actually really easy. Students love pronunciation bingo when they know how to play it. It is great fun.

By providing game as a media in teaching pronunciation to intermediate school students, I hope that it will make them fell something pleasant and fell it different from what they use to get in the class. The use of game will stimulate them to be more active and it is really match with the students' world where pre-level 
students liked to study through movement. By the activity, they will give an opportunity to express their mind, emotion, feeling and attitudes when they involve in it. Reviewed at the explanation above, I assume that the use of game is one of good and effective ways to teach and to motivate them as young learners to study.

\section{METHOD}

The writer take the true - experiment research as one of the instrument used. According to Nunan that true-experiment has both pre - and posttest, experimental and control group, and random assignment of the subjects. On the other hand, quantitative research is prominent and reliable, objective, generalizable, results oriented, and assumes the existence of facts that are somehow outside and are not dependent on the observer or researcher (Nunan, 1992).

Its means that writer uses this method to analyze the collected data from the field and must go to the place of research, while to test using the pronunciation bingo game. In this research there are two variables used there are: The use of Pronunciation Bingo Game as a variable $\mathrm{X}$ while Improve Students' Pronunciation as variable $Y$.

\section{DISCUSSION}

Data of the research are taken from the first grade student of MTs Al-
Khairiyah Kepandean Ciruas, with a total of population 193 students. In this research paper, the writer takes 60 students as the sample. In this discussion, the writer will explain the result of investigation using statistical analysis.

\section{Test of Normality}

a. Test Normality of Experiment Class

To investigate student's worksheet gives and describes score in table with formula:

\section{Student's Final Score $=$}

$\frac{\text { Student's Raw Score }}{\text { Ideal Maximum Score }(30)} \times 100$

Based on the table above, the writer will arrange one by one from the lowest until high score from that table, as follows:

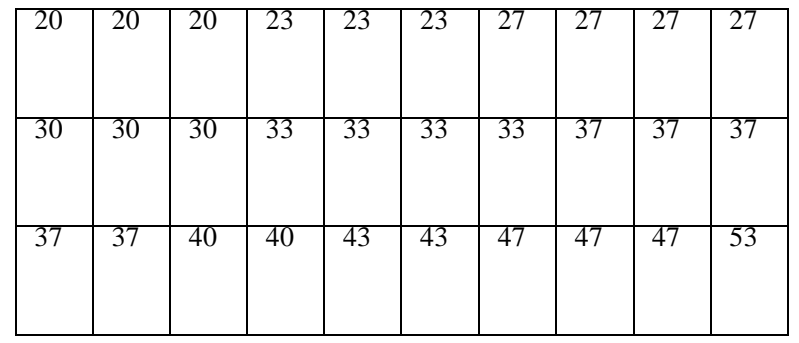

1. Find out range, with formula

$$
\begin{aligned}
R & =(H-L) \\
& =(53-20) \\
& =33
\end{aligned}
$$

2. Looking for the class interval, with formula:

$$
\begin{aligned}
K & =1+3,3(\log n) \\
& =1+3,3(\log .30)
\end{aligned}
$$


Volume 2 No. 2, Agustus 2019

Universitas Banten Jaya

$$
\begin{aligned}
& =1+3,3(1,48) \\
& =1+4,88 \\
& =5,88=6 \text { (become) }
\end{aligned}
$$

3. Looking for the long class, with formula:

$$
\begin{aligned}
& P=\frac{R}{K} \\
& =\frac{33}{6} \\
& =5,5=6 \text { (become) }
\end{aligned}
$$

4. Looking for mean

$$
\begin{aligned}
\bar{X} & =\frac{\sum F X}{\sum F}=\frac{999}{30} \\
& =33,3
\end{aligned}
$$

5. Looking for median

$$
\begin{aligned}
M e & =b+p\left\{\frac{1 / 2 N-F k a}{F}\right\} \\
& =37,5+6\left\{\frac{1 / 215-13}{9}\right\} \\
& =37,5+6\left\{\frac{2}{9}\right\} \\
& =37,5+6(0,22) \\
& =37,5+1,32 \\
& =38,82
\end{aligned}
$$

6. Looking for mode

$$
\begin{aligned}
\text { Mo } & =\mathrm{b}+\mathrm{p}\left[\frac{b i}{b 1+b 2}\right] \\
& =37.5+6\left[\frac{9-7}{(9-7)+(9-4)}\right] \\
& =37.5+6\left[\frac{2}{2+5}\right]
\end{aligned}
$$

$$
\begin{aligned}
& =37.5+6(0,28) \\
& =37,5+1,68 \\
& =39,18
\end{aligned}
$$

7. Looking for mode

$$
\begin{aligned}
\text { Mo } & =\mathrm{b}+\mathrm{p}\left[\frac{b i}{b 1+b 2}\right] \\
& =37.5+6\left[\frac{9-7}{(9-7)+(9-4)}\right] \\
& =37.5+6\left[\frac{2}{2+5}\right] \\
& =37.5+6(0,28) \\
& =37,5+1,68 \\
& =39,18
\end{aligned}
$$

8. Making assist table deviation standard

\section{Table 4.3}

Assist Table Deviation Standard Pretest in

\section{Experiment Class}

\begin{tabular}{|l|l|l|l|l|l|l|}
\hline $\begin{array}{l}\text { Interv } \\
\text { al }\end{array}$ & $\mathrm{F}$ & $\mathrm{X}$ & $\mathrm{FX}$ & $\begin{array}{l}- \\
\bar{X}\end{array}$ & $\left.\begin{array}{l}(\mathrm{X}- \\
\bar{X}\end{array}\right)$ & $\begin{array}{l}\mathrm{F}(\mathrm{X}- \\
\bar{X}) 2\end{array}$ \\
\hline $20-$ & 6 & 22, & 135 & - & 116, & 699,8 \\
25 & 7 & 5 & 199 & 10, & 64 & 4 \\
31 & 9 & $\begin{array}{l}28, \\
26-\end{array}$ & 5 & 8 & 23,0 & 161,2 \\
$32-$ & 4 & 34, &, 5 & 4,8 & 1,44 & 12,96 \\
37 & 3 & 5 & 162 & 1,2 & 51,8 & 207,3 \\
$38-$ & & 40, & & & & \\
\hline
\end{tabular}


Volume 2 No. 2, Agustus 2019

P-ISSN 2623-0062

Universitas Banten Jaya

E-ISSN 2622-9056

\begin{tabular}{|l|l|l|l|l|l|l|}
\hline 43 & 1 & 5 & 139 & 7,2 & 4 & 6 \\
$44-$ & & 46, &, 5 & 13, & 174, & 522,7 \\
49 & & 5 & 52, & 2 & 24 & 2 \\
$50-$ & & 52, & 5 & 19, & 364, & 368,6 \\
55 & & 5 & & 2 & 64 & 44 \\
\hline$\sum$ & $\mathbf{3}$ & & $\mathbf{9 9 9}$ & & & $\mathbf{1 9 7 2 ,}$ \\
& $\mathbf{0}$ & & & & & $\mathbf{8}$ \\
\hline
\end{tabular}

a. Accounting deviation standard

$$
\begin{aligned}
S D & =\sqrt{\frac{\sum f(x-\bar{x})^{2}}{\mathrm{~N}}} \\
& =\sqrt{\frac{1972,8}{30}} \\
& =\sqrt{65,76} \\
& =8,10
\end{aligned}
$$

\begin{tabular}{|c|c|c|c|c|c|c|}
\hline $\begin{array}{c}26- \\
31\end{array}$ & & & & $\begin{array}{c}0.24 \\
44\end{array}$ & $\begin{array}{c}7,3 \\
3\end{array}$ & 7 \\
\hline & $\begin{array}{c}31 \\
5\end{array}$ & $-0,22$ & $\begin{array}{c}0,41 \\
29\end{array}$ & & & \\
\hline $\begin{array}{c}32- \\
37\end{array}$ & & & & $\begin{array}{c}0,28 \\
21\end{array}$ & $\begin{array}{c}8,4 \\
6\end{array}$ & 9 \\
\hline & $\begin{array}{c}37 \\
5\end{array}$ & 0,51 & $\begin{array}{c}0,69 \\
5\end{array}$ & & & \\
\hline $\begin{array}{c}38- \\
43\end{array}$ & & & & $\begin{array}{c}0,19 \\
94\end{array}$ & $\begin{array}{c}5,9 \\
8\end{array}$ & 4 \\
\hline & $\begin{array}{c}43 . \\
5\end{array}$ & 1,25 & $\begin{array}{r}0,89 \\
44\end{array}$ & & & \\
\hline $\begin{array}{c}44- \\
49\end{array}$ & & & & $\begin{array}{c}0,08 \\
28\end{array}$ & $\begin{array}{c}2,4 \\
8\end{array}$ & 3 \\
\hline & $\begin{array}{c}49 \\
5\end{array}$ & 2,00 & $\begin{array}{r}0,97 \\
72\end{array}$ & & & \\
\hline $\begin{array}{c}50- \\
55\end{array}$ & & & & $\begin{array}{c}0,01 \\
97\end{array}$ & $\begin{array}{c}0,5 \\
9\end{array}$ & 1 \\
\hline & $\begin{array}{c}55 \\
5\end{array}$ & 2,74 & $\begin{array}{c}0,99 \\
69\end{array}$ & & & \\
\hline
\end{tabular}

b. Test normality of Pre-test in Experiment Class

Table 4.4

Test of Normality with Chi Square $\left(\mathrm{X}^{2}\right)$

\begin{tabular}{|l|l|c|c|c|c|c|}
\hline Interv & Clas & $\mathbf{Z}_{\text {acco }}$ & $\mathbf{Z}_{\text {tabl }}$ & $\mathbf{L z}_{\text {tabl }}$ & $\mathbf{E}_{\mathbf{i}}$ & $\mathbf{O}$ \\
$\mathbf{S}$ & unt & e & $e$ & & $\mathbf{i}$ \\
& Limi & & & & & \\
$\mathbf{t}$ & & & & & \\
\hline $20-$ & 19, & -1.70 & 0.04 & & & \\
\hline 25 & 5 & & 46 & & & \\
\hline & 25, & -0.96 & 0.16 & & & \\
& 5 & & 85 & & & \\
\hline
\end{tabular}

c. Accounting $X^{2}$ (chi square), with formula:

$$
\mathrm{X}_{\text {account }}^{2} \sum \frac{(O i-E i)^{2}}{E i}
$$

$\frac{(6-3,71)^{2}}{3,71}+\frac{(7-7,33)^{2}}{7,33}+\frac{(9-8,46)^{2}}{8,46}+\frac{(4-5,98)^{2}}{5,98}+\frac{(3-2,48)^{2}}{2,48}+\frac{(1-0,59)^{2}}{0,59}$

$=1,41+0,01+0,03+0,65+0,10+0,28$

$=2,48$

d. Looking for degree of freedom (df), with formula:

$$
\begin{aligned}
\mathrm{Df} & =\mathrm{k}-3 \\
& =6-3
\end{aligned}
$$


Volume 2 No. 2, Agustus 2019

$$
=3
$$

e. Determining $\mathrm{X}_{\text {table }}^{2}$ with signification $5 \%$ and $\mathrm{df}(3)$

$$
\begin{aligned}
\mathrm{X}_{\text {table }}^{2} & =(1-\alpha)(\mathrm{df}) \\
& =(1-0,05)(3) \\
& =(0,95)(3) \\
& =7.81
\end{aligned}
$$

Based on the calculation above is known that $X_{\text {account }}^{2}=2,48$ and $X_{\text {table }}^{2}=$ 7,81 so $X_{\text {account }}^{2}=2,48<X_{\text {table }}^{2}=7,81$. The concluded that the test (sample) of the population is normal distribution.

\section{The Result of Post-test in Experiment}

\section{Class}

\begin{tabular}{|l|l|l|l|l|l|l|l|l|l|}
\hline 5 & 6 & 6 & 7 & 7 & 7 & 73 & 73 & 73 & 73 \\
3 & 3 & 7 & 0 & 0 & 0 & & & & \\
\hline 7 & 7 & 7 & 8 & 8 & 8 & 83 & 83 & 83 & 83 \\
7 & 7 & 7 & 0 & 0 & 0 & & & & \\
\hline 8 & 8 & 8 & 9 & 9 & 9 & 10 & 10 & 10 & 10 \\
7 & 7 & 7 & 0 & 0 & 0 & 0 & 0 & 0 & 0 \\
\hline
\end{tabular}

1. Find out range, with formula

$$
\begin{aligned}
\mathrm{R} & =\mathrm{H}-\mathrm{L} \\
& =100-53=47
\end{aligned}
$$

2. Looking for the class interval, with formula:

$$
\begin{aligned}
\mathrm{K} & =1+3,3(\log \mathrm{n}) \\
& =1+3,3(\log .30) \\
& =1+3,3(1,48) \\
& =1+4,88 \\
& =5,88=6 \text { (become) }
\end{aligned}
$$

3. Looking for the long class, with formula:

$$
p=\frac{R}{K}=\frac{47}{6}=7,83=8 \text { (become) }
$$

4. Making distribution frequency table

Table 4.5

Distribution Frequency

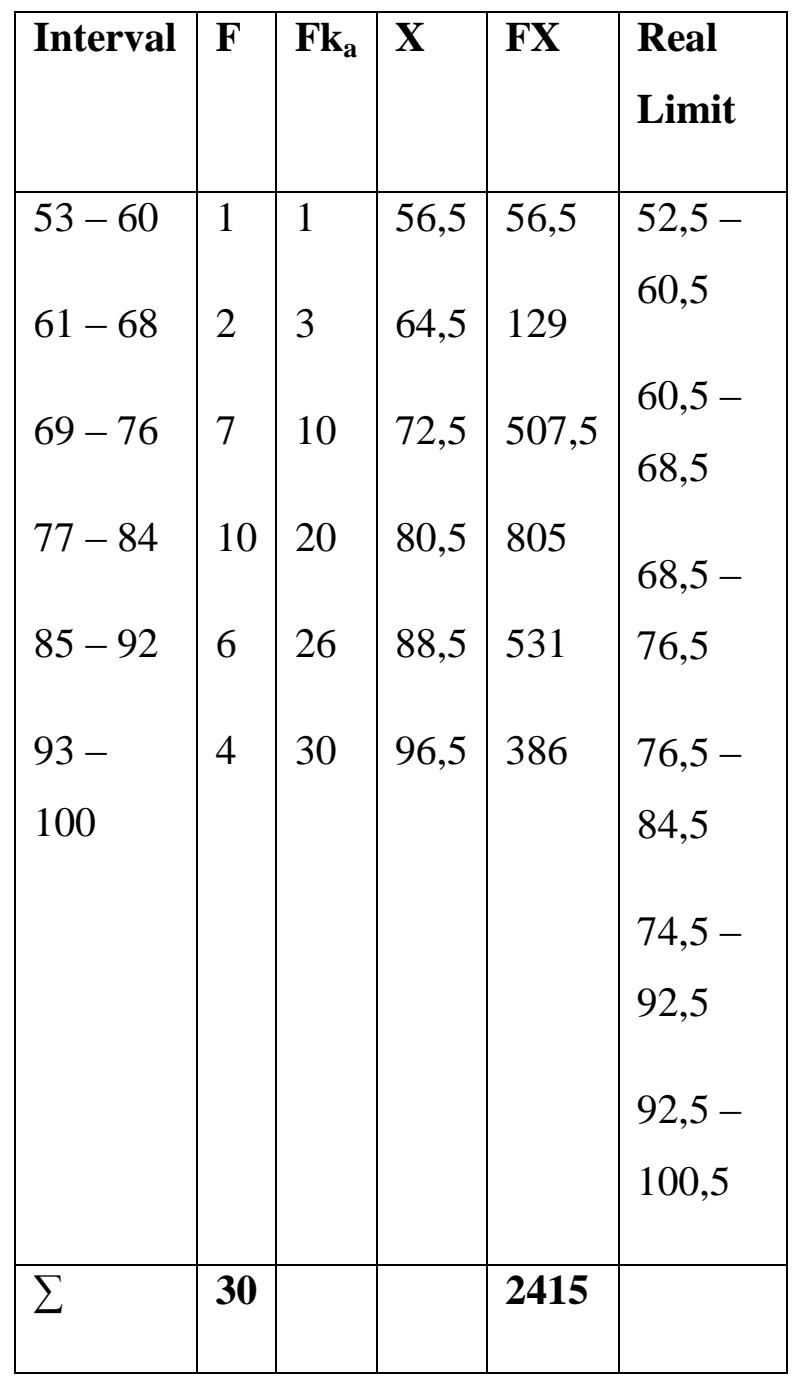


Volume 2 No. 2, Agustus 2019

Universitas Banten Jaya

5. Histogram and Polygon Graph

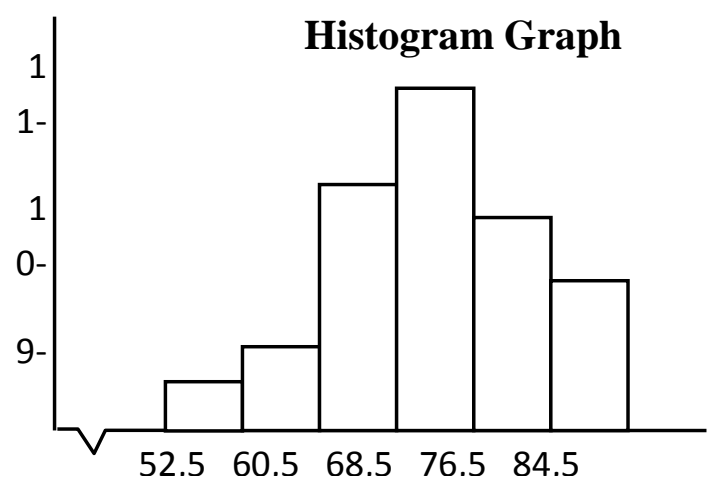

Polygon Graph

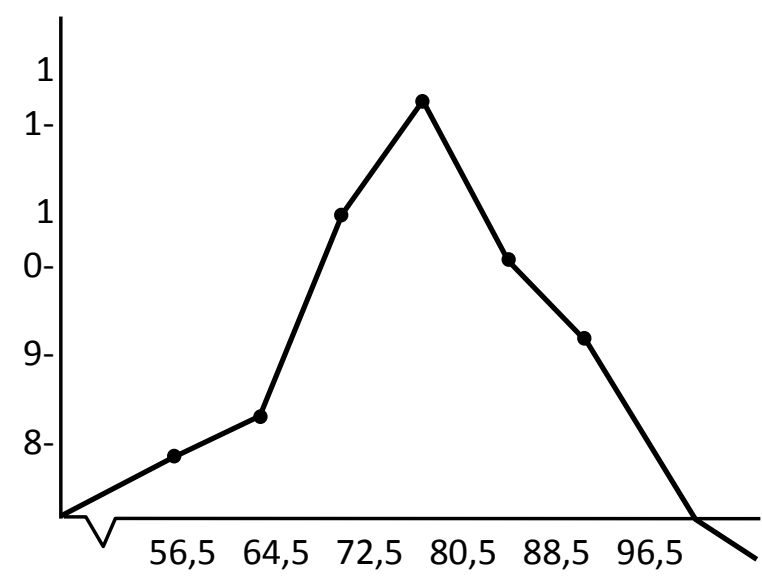

6. Looking for mean
P-ISSN 2623-0062

E-ISSN 2622-9056

\begin{tabular}{|c|c|c|c|c|c|c|}
\hline $\begin{array}{c}\text { Inter } \\
\text { val }\end{array}$ & $\begin{array}{l}\text { Class } \\
\text { Limit }\end{array}$ & $\begin{array}{c}\mathbf{Z}_{\text {acco }} \\
\text { unt }\end{array}$ & $\mathbf{Z}_{\text {table }}$ & $\begin{array}{c}\mathbf{L z}_{t a b} \\
l e\end{array}$ & $\mathbf{E}_{\mathbf{i}}$ & $\begin{array}{l}\mathbf{O} \\
\mathbf{i}\end{array}$ \\
\hline & 52,5 & -282 & $\begin{array}{c}0,00 \\
24\end{array}$ & & & \\
\hline $\begin{array}{c}53- \\
60\end{array}$ & & & & $\begin{array}{c}0,01 \\
93\end{array}$ & $\begin{array}{c}0,5 \\
7\end{array}$ & 1 \\
\hline & 60,5 & $-2,02$ & $\begin{array}{c}0,02 \\
17\end{array}$ & & & \\
\hline $\begin{array}{c}61- \\
68\end{array}$ & & & & $\begin{array}{c}0,09 \\
14\end{array}$ & $\begin{array}{c}2,7 \\
4\end{array}$ & 2 \\
\hline & 68,5 & $-1,21$ & $\begin{array}{c}0,11 \\
31\end{array}$ & & & \\
\hline $\begin{array}{c}69- \\
76\end{array}$ & & & & $\begin{array}{c}0,23 \\
15\end{array}$ & $\begin{array}{c}6,9 \\
4\end{array}$ & 7 \\
\hline & 76,5 & $-0,40$ & $\begin{array}{c}0,34 \\
46\end{array}$ & & & \\
\hline $\begin{array}{c}77- \\
84\end{array}$ & & & & $\begin{array}{c}0,31 \\
08\end{array}$ & $\begin{array}{c}9,3 \\
2\end{array}$ & 0 \\
\hline & 84,5 & 0,40 & $\begin{array}{c}0,65 \\
54\end{array}$ & & & \\
\hline $\begin{array}{c}85- \\
92\end{array}$ & & & & $\begin{array}{c}0,23 \\
15\end{array}$ & $\begin{array}{c}6,9 \\
4\end{array}$ & 6 \\
\hline & 92,5 & 1,21 & $\begin{array}{c}0,88 \\
69\end{array}$ & & & \\
\hline $\begin{array}{l}93- \\
100\end{array}$ & & & & $\begin{array}{c}0,09 \\
14\end{array}$ & $\begin{array}{c}2,7 \\
4\end{array}$ & 4 \\
\hline & 100,5 & 2,02 & $\begin{array}{c}0,97 \\
83\end{array}$ & & & \\
\hline
\end{tabular}

7. Looking for median 
Volume 2 No. 2, Agustus 2019

P-ISSN 2623-0062

Universitas Banten Jaya

E-ISSN 2622-9056

$$
\begin{aligned}
M e= & b+p\left\{\frac{1 / 2^{N-F k a}}{F}\right\} \\
& =84,5+6\left\{\frac{15-10}{10}\right\} \\
& =84,5+6\left\{\frac{5}{10}\right\} \\
& =84,5+6(0,5) \\
& =84,5+3 \\
& =87,5
\end{aligned}
$$

\begin{tabular}{|ll|l|l|l|l|l|l|}
\hline 93 & 4 & 96,5 & 386 & 16 & 256 & 1024 \\
100 & & & & & & \\
\hline$\sum$ & $\mathbf{3 0}$ & & $\mathbf{2 4 1 5}$ & & & $\mathbf{2 9 4 4}$ \\
\hline
\end{tabular}

a. Accounting deviation standard

8. Looking for mode

$$
\begin{aligned}
\text { Mo } & =\mathrm{b}+\mathrm{p}\left[\frac{b i}{b 1+b 2}\right] \\
& =84,5+6\left[\frac{10-7}{(10-7)+(10-6)}\right] \\
& =84.5+6\left[\frac{3}{3+4}\right] \\
& =84.5+6(0,42) \\
& =84,5+2,52 \\
& =87,02
\end{aligned}
$$

\begin{tabular}{|c|c|c|c|c|c|c|c|}
\hline Interval & $\mathbf{F}$ & $\mathbf{X}$ & FX & $\begin{array}{l}(\mathrm{X}- \\
\bar{X})\end{array}$ & $\begin{array}{l}(\mathrm{X}- \\
\bar{X})^{2}\end{array}$ & $\begin{array}{l}\mathbf{F}(\mathbf{X}- \\
\bar{X})^{2}\end{array}$ & $\begin{array}{l}\text { c. Accounting } X^{2} \text { (chi square), with } \\
\text { formula: }\end{array}$ \\
\hline $53-60$ & 1 & 56,5 & 56,5 & -24 & 576 & 576 & \\
\hline $61-68$ & 2 & 64,5 & 129 & -16 & 256 & 512 & $\mathrm{X}_{\text {account }}^{2}=\sum \frac{(O i-E i)^{2}}{E i}$ \\
\hline $69-76$ & 7 & 72,5 & 507,5 & -8 & 64 & 448 & $\begin{array}{l}= \\
(1-0,57)^{2} \\
\frac{(2-2,74)^{2}}{+}+\frac{(7-6,94)^{2}}{+(10-9,32)^{2}}+\frac{(6-6,94)^{2}}{2}+\frac{(4-2,74)^{2}}{}\end{array}$ \\
\hline $77-84$ & 10 & 80,5 & 805 & 0 & 0 & 0 & 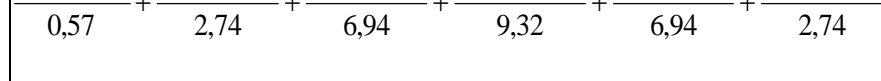 \\
\hline $85-92$ & 6 & 88,5 & 531 & 8 & 64 & 384 & $\begin{array}{l}=0,32+0,19+0,01+0,04+0,12+0,57 \\
=1,25\end{array}$ \\
\hline
\end{tabular}

9. Making assist table deviation standard

Table 4.6

b. Test normality of Pre-test in Experiment Class

Table. 4.7

Assist Table Deviation Standard Pre-test in Experiment Class

\section{Test of Normality with Chi Square $\left(\mathbf{X}^{2}\right)$}


d. Looking for degree of freedom (df), with formula:

$\mathrm{Df}=\mathrm{k}-3$

$=6-3$

$=3$

e. Determining $\quad X_{\text {table }}^{2}$ with signification 5\% and df (3)

$$
\begin{aligned}
\mathrm{X}_{\text {table }}^{2} & =(1-\alpha)(\mathrm{df}) \\
& =(1-0,05)(3) \\
& =(0,95)(3) \\
& =7.81
\end{aligned}
$$

Based on the calculation above is known that $\mathrm{X}_{\text {account }}=1,25$ and $\mathrm{X}_{\text {table }}^{2}=$ 7,81 so $X_{\text {account }}^{2}=1,25<X_{\text {table }}^{2}=7,81$. The concluded that the test (sample) of the population is normal distribution.

\section{b. Test Normality of Control Class}

To investigate student's worksheet gives and describes score in table with formula:

Student's Final Score $=$ $\frac{\text { Student's Raw Score }}{\text { Ideal Maximum Score }(30)} \times 100$

\begin{tabular}{|c|c|c|c|}
\hline \multirow{2}{*}{ No } & \multirow{2}{*}{ Name } & \multicolumn{2}{|l|}{ Score } \\
\hline & & Pre-test & Post-test \\
\hline 1 & AZA & 23 & 27 \\
\hline 2 & AS & 50 & 33 \\
\hline 3 & DM & 37 & 37 \\
\hline 4 & Fdl & 30 & 20 \\
\hline 5 & Fjr & 43 & 30 \\
\hline 6 & FK & 27 & 23 \\
\hline 7 & Frm & 30 & 33 \\
\hline 8 & Hsn & 30 & 33 \\
\hline 9 & $\mathrm{IH}$ & 33 & 30 \\
\hline 10 & IA & 37 & 40 \\
\hline 11 & LH & 20 & 33 \\
\hline 12 & Lsy & 37 & 20 \\
\hline 13 & Mht & 33 & 43 \\
\hline 14 & Mrf & 27 & 23 \\
\hline 15 & Mgw & 17 & 17 \\
\hline 16 & Mua & 23 & 20 \\
\hline 17 & Mnwr & 23 & 27 \\
\hline 18 & $\mathrm{Njm}$ & 27 & 23 \\
\hline 19 & Rhmt & 20 & 30 \\
\hline 20 & Rst & 23 & 30 \\
\hline
\end{tabular}

Table 4.8

\section{Result of Pre-test and Post-test in} Control Class 
Volume 2 No. 2, Agustus 2019

Universitas Banten Jaya

\begin{tabular}{|l|l|l|l|}
\hline 21 & Seperti & 17 & 17 \\
\hline 22 & SG & 20 & 40 \\
\hline 23 & SK & 33 & 20 \\
\hline 24 & SS & 30 & 27 \\
\hline 25 & Shr & 17 & 17 \\
\hline 26 & Sry & 33 & 50 \\
\hline 27 & Sym & 30 & 23 \\
\hline 28 & TF & 20 & 27 \\
\hline 29 & TNJ & 33 & 33 \\
\hline 30 & Ikls & 23 & 43 \\
\hline
\end{tabular}

Based on the table above, the writer will arrange one by one from the lowest until high score from that table, as follows:

\section{The Result of Pre-test in Control Class}

\begin{tabular}{|l|l|l|l|l|l|l|l|l|l|}
\hline 1 & 1 & 1 & 2 & 2 & 2 & 2 & 2 & 2 & 2 \\
7 & 7 & 7 & 0 & 0 & 0 & 0 & 3 & 3 & 3 \\
\hline 2 & 2 & 2 & 2 & 2 & 3 & 3 & 3 & 3 & 3 \\
3 & 3 & 7 & 7 & 7 & 0 & 0 & 0 & 0 & 0 \\
\hline 3 & 3 & 3 & 3 & 3 & 3 & 3 & 3 & 4 & 5 \\
3 & 3 & 3 & 3 & 3 & 7 & 7 & 7 & 3 & 0 \\
\hline
\end{tabular}

1. Find out range, with formula

$$
\begin{aligned}
\mathrm{R} & =\mathrm{H}-\mathrm{L} \\
& =50-17 \\
& =33
\end{aligned}
$$

P-ISSN 2623-0062

E-ISSN 2622-9056

2. Looking for the class interval, with formula:

$$
\begin{aligned}
\mathrm{K} & =1+3,3(\log \mathrm{n}) \\
& =1+3,3(\log .30) \\
& =1+3,3(1,48) \\
& =1+4,88 \\
& =5,88=6 \text { (become) }
\end{aligned}
$$

3. Looking for the long class, with formula:

$$
\begin{aligned}
p & =\frac{R}{K} \\
& =\frac{33}{6} \\
& =5,5=6 \text { (become) }
\end{aligned}
$$

4. Making distribution frequency table

Table 4.9

\section{Distribution Frequency}

\begin{tabular}{|l|l|l|l|l|l|}
\hline Interval & $\mathbf{F}$ & $\mathbf{F k}_{\mathbf{a}}$ & $\mathbf{X}$ & $\mathbf{F X}$ & $\begin{array}{l}\text { Real } \\
\text { Limit }\end{array}$ \\
\hline $17-22$ & 7 & 7 & 19,5 & 136,5 & $\begin{array}{l}16,5- \\
22,5\end{array}$ \\
$29-28$ & 8 & 15 & 25,5 & 204 & $22,5-$ \\
$35-40$ & 3 & 28 & 37,5 & 112,5 & $28,5-$ \\
$41-46$ & 1 & 29 & 43,5 & 43,5 & 34,5 \\
$47-52$ & 1 & 30 & 49,5 & 49,5 & $34,5-$ \\
& & & & & 40,5 \\
& & & & & $40,5-$ \\
\hline
\end{tabular}




\begin{tabular}{|l|l|l|l|l|l|}
\hline & & & & & 46,5 \\
& & & & & $46,5-$ \\
52,5
\end{tabular}

5. Histogram and Polygon Graph

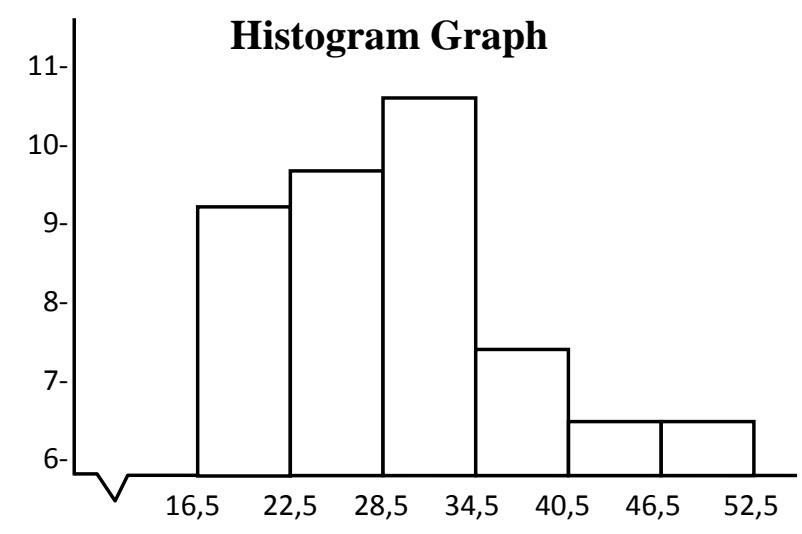

Polygon Graph

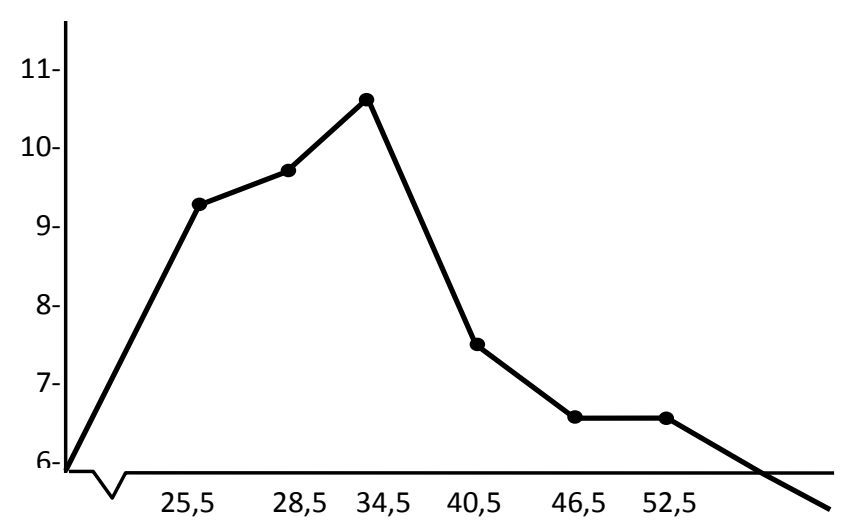

7. Looking for median

$$
\begin{aligned}
M e & =b+p\left\{\frac{1 / 2 N-F k a}{F}\right\} \\
& =34,5+6\left\{\frac{15-15}{10}\right\} \\
& =34,5+6\left\{\frac{0}{10}\right\} \\
& =34,5+6(0) \\
& =34,5+0 \\
& =34,5
\end{aligned}
$$

8. Looking for mode

$$
\begin{aligned}
\text { Mo } & =\mathrm{b}+\mathrm{p}\left[\frac{b i}{b 1+b 2}\right] \\
& =34,5+6\left[\frac{10-8}{(10-8)+(10-3)}\right] \\
& =34,5+6\left[\frac{2}{2+7}\right] \\
& =34,5+6(0,22) \\
& =34,5+1,32 \\
& =35,82
\end{aligned}
$$

9. Making assist table deviation standard

Table 4.10

Assist Table Deviation Standard Pretest in Experiment Class

6. Looking for mean

$$
\begin{aligned}
\bar{X} & =\frac{\sum F X}{\sum F}=\frac{861}{30} \\
& =28,7
\end{aligned}
$$

\begin{tabular}{|l|l|l|l|l|l|l|}
\hline Interv & F & $\mathbf{X}$ & $\mathbf{F X}$ & $(\mathbf{X}$ & $(\mathbf{X}-$ & F(X- \\
al & & & & - & $\bar{X})^{2}$ & $\bar{X})^{2}$ \\
& & & & $\bar{X}$ & & \\
& & & & & & \\
\hline
\end{tabular}




\begin{tabular}{|c|c|c|c|c|c|c|c|c|c|c|c|c|c|}
\hline $\begin{array}{l}17- \\
22 \\
23- \\
28\end{array}$ & 1 & $\begin{array}{l}19, \\
5 \\
25, \\
5\end{array}$ & $\begin{array}{l}136, \\
5 \\
204\end{array}$ & $\begin{array}{l}- \\
3,2\end{array}$ & $\begin{array}{l}84,6 \\
4 \\
10,2 \\
4\end{array}$ & $\begin{array}{l}592, \\
48 \\
81,9 \\
2\end{array}$ & \multicolumn{7}{|c|}{$\begin{array}{l}\text { Table } 4.11 \\
\text { Test of Normality with Chi Square }\left(X^{2}\right)\end{array}$} \\
\hline $\begin{array}{l}29- \\
34\end{array}$ & 3 & $\begin{array}{l}31, \\
5\end{array}$ & $\begin{array}{l}112, \\
5\end{array}$ & 2,8 & 7,84 & \multicolumn{2}{|c|}{78 , Interval } & $\begin{array}{l}\text { Class } \\
\text { Limit }\end{array}$ & $\mathbf{Z}_{\text {account }}$ & $\mathbf{Z}_{\text {table }}$ & $\mathbf{L z}_{\text {table }}$ & $\mathbf{E}_{\mathbf{i}}$ & $\mathbf{O}_{\mathbf{i}}$ \\
\hline $\begin{array}{l}35- \\
40\end{array}$ & 1 & $\begin{array}{l}37, \\
5\end{array}$ & 43,5 & 14 & 4 & 32 & -22 & 16,5 & $-1,65$ & 0,0495 & 0,1509 & 4,52 & 7 \\
\hline $41-$ & & 43 & 49,5 & 8 & $\begin{array}{l}219, \\
04\end{array}$ & $\begin{array}{l}219, \\
0423\end{array}$ & -28 & 22,5 & $-0,84$ & 0,2004 & 0,2916 & 8,74 & 8 \\
\hline 46 & & 5 & & 8 & 432 & 432 & & 28,5 & $-0,02$ & 0,492 & & & \\
\hline \multirow{3}{*}{$\begin{array}{l}47- \\
52\end{array}$} & & \multirow{3}{*}{$\begin{array}{l}49, \\
5\end{array}$} & & & 64 & $64^{29}$ & -34 & & & & 0,2903 & 8,70 & 10 \\
\hline & & & & & & & & 34,5 & 0,78 & 0,7823 & & & \\
\hline & & & & & & 35 & -40 & & & & 0,1618 & 4,85 & 3 \\
\hline \multirow[t]{2}{*}{$\sum$} & 3 & & 861 & & & 1636 & & 40,5 & 1,59 & 0,9441 & & & \\
\hline & 0 & & & & & & -46 & & & & 0,0479 & 1,43 & 1 \\
\hline & & & & & & & & 46,5 & 2,41 & 0,992 & & & \\
\hline & & & & & & & -52 & & & & 0,0074 & 0,22 & 1 \\
\hline & $A$ & cou & $\operatorname{ing~} \mathrm{c}$ & . & stan & & & 52,5 & 3,22 & 0,9994 & & & \\
\hline
\end{tabular}

$$
\begin{aligned}
S D & =\sqrt{\frac{\sum f(x-\bar{x})^{2}}{\mathrm{~N}}} \\
& =\sqrt{\frac{1636,8}{30}} \\
& =\sqrt{54,56} \\
& =7,38
\end{aligned}
$$

c. Accounting $X^{2}$ (chi square), with formula:

$$
\mathrm{X}_{\text {account }}^{2}=\sum \frac{(O i-E i)^{2}}{E i}
$$$$
=
$$$$
\frac{(7-4,52)^{2}}{4,52}+\frac{(8-8,74)^{2}}{8,74}+\frac{(10-8,70)^{2}}{8,70}+\frac{(3-4,85)^{2}}{4,85}+\frac{(1-1,43)^{2}}{1,43}+\frac{(1-0,22)^{2}}{0,22}
$$

b. Test normality of Pre-test in Control Class

$$
\begin{aligned}
& =1,36+0,06+0,19+0,70+0,12 \\
& +2,76=5,19
\end{aligned}
$$

d. Looking for degree of freedom (df), with formula: 
Volume 2 No. 2, Agustus 2019

Universitas Banten Jaya

$$
\begin{aligned}
\text { Df } & =k-3 \\
& =6-3 \\
& =3
\end{aligned}
$$

e. Determining $X_{\text {table }}^{2}$

with signification $5 \%$ and $\mathrm{df}(3)$

$$
\begin{aligned}
X_{\text {table }}^{2} & =(1-\alpha)(\mathrm{df}) \\
= & (1-0,05)(3) \\
= & (0,95)(3) \\
= & 7.81
\end{aligned}
$$

Based on the calculation above is known that $\mathrm{X}^{2}$ account $=2,48$ and $\mathrm{X}_{\text {table }}^{2}=$ 7,81 so $X_{\text {account }}^{2}=5,19<X_{\text {table }}^{2}=7,81$. The concluded that the test (sample) of the population is normal distribution.

\section{The Result of Post-test in Control Class}

\begin{tabular}{|l|l|l|l|l|l|l|l|l|l|}
\hline 1 & 1 & 1 & 2 & 2 & 2 & 2 & 2 & 2 & 2 \\
7 & 7 & 7 & 0 & 0 & 0 & 0 & 3 & 3 & 3 \\
\hline 2 & 2 & 2 & 2 & 2 & 3 & 3 & 3 & 3 & 3 \\
3 & 7 & 7 & 7 & 7 & 0 & 0 & 0 & 0 & 3 \\
\hline 3 & 3 & 3 & 3 & 3 & 4 & 4 & 4 & 4 & 5 \\
3 & 3 & 3 & 3 & 7 & 0 & 0 & 3 & 3 & 0 \\
\hline
\end{tabular}

1. Find out range, with formula

$$
\begin{aligned}
\mathrm{R} & =\mathrm{H}-\mathrm{L} \\
& =50-17 \\
& =33
\end{aligned}
$$

2. Looking for the class interval, with formula:

$$
\mathrm{K}=1+3,3(\log \mathrm{n})
$$

$$
\begin{aligned}
& =1+3,3(\log .30) \\
& =1+3,3(1,48) \\
& =1+4,88 \\
& =5,88=6 \text { (become) }
\end{aligned}
$$

3. Looking for the long class, with formula:

$$
\begin{aligned}
p & =\frac{R}{K} \\
& =\frac{33}{6} \\
& =5,5 \\
& =6 \text { (become) }
\end{aligned}
$$

4. Making distribution freuquency table

Table 4.12

\section{Distribution Frequency}

\begin{tabular}{|l|l|l|l|l|l|}
\hline Interval & $\mathbf{F}$ & $\mathbf{F k}_{\mathbf{a}}$ & $\mathbf{X}$ & $\mathbf{F X}$ & $\begin{array}{l}\text { Real } \\
\text { Limit }\end{array}$ \\
\hline $17-22$ & 7 & 7 & 19,5 & 136,5 & $16,5-$ \\
$23-28$ & 8 & 15 & 25,5 & 204 & 22,5 \\
$29-34$ & 9 & 24 & 31,5 & 283,5 & $22,5-$ \\
$35-40$ & 3 & 27 & 37,5 & 112,5 & 28,5 \\
$41-46$ & 2 & 29 & 43,5 & 87 & 34,5 \\
\hline
\end{tabular}


Volume 2 No. 2, Agustus 2019

P-ISSN 2623-0062

Universitas Banten Jaya

E-ISSN 2622-9056

\begin{tabular}{|l|l|l|l|l|l|}
\hline $47-52$ & 1 & 30 & 49,5 & 49,5 & $34,5-$ \\
& & & & & 40,5 \\
& & & & & $40,5-$ \\
& & & & & 46,5 \\
& & & & & $46,5-$ \\
& & & & & 52,5 \\
\hline$\sum$ & $\mathbf{3 0}$ & & & $\mathbf{8 7 3}$ & \\
\hline
\end{tabular}

6. Looking for mean

$\bar{X}=\frac{\sum F X}{\sum F}$

$=\frac{861}{30}$

$=28,7$

7. Looking for median

$$
\begin{aligned}
M e= & b+p\left\{\frac{1 / 2 N-F k a}{F}\right\} \\
& =34,5+6\left\{\frac{15-5}{9}\right\} \\
& =34,5+6\left\{\frac{0}{10}\right\} \\
& =34,5+6(0) \\
& =34+0 \\
& =34,5
\end{aligned}
$$

8. Looking for mode

$$
\begin{aligned}
\text { Mo } & =\mathrm{b}+\mathrm{p}\left[\frac{b i}{b 1+b 2}\right] \\
& =34,5+6\left[\frac{9-8}{(9-8)+(9-3)}\right] \\
& =34,5+6\left[\frac{1}{1+6}\right]
\end{aligned}
$$

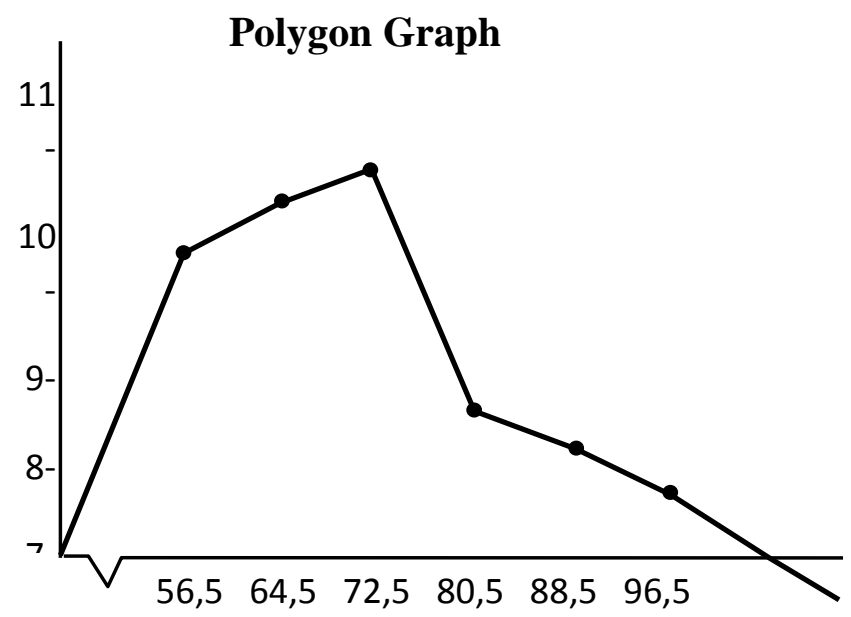

$$
\begin{aligned}
& =34,5+6\left[\frac{1}{7}\right] \\
& =34,5+6(0,14) \\
& =34,5+0,84=35,34
\end{aligned}
$$

9. Making assist table deviation standard 
Volume 2 No. 2, Agustus 2019

P-ISSN 2623-0062

Universitas Banten Jaya

E-ISSN 2622-9056

Table 4.13

Assist Table Deviation Standard

Post-test in Control Class b. Test normality of Post-test in Control Class

\begin{tabular}{|c|c|c|c|c|c|c|c|c|c|c|c|c|c|}
\hline \multirow[t]{2}{*}{$\begin{array}{l}\text { Interv } \\
\text { al }\end{array}$} & \multirow[t]{2}{*}{$\mathbf{F}$} & \multirow[t]{2}{*}{$\mathbf{X}$} & \multirow[t]{2}{*}{ FX } & \multirow{2}{*}{$\begin{array}{l}\mathbf{X} \\
- \\
\bar{X} \\
{ }^{2}\end{array}$} & \multirow[t]{2}{*}{$\begin{array}{l}(\mathrm{X}- \\
\bar{X})^{2}\end{array}$} & $\begin{array}{l}\mathbf{F}(\mathbf{X}- \\
\bar{X})^{2}\end{array}$ & \multicolumn{7}{|c|}{$\begin{array}{l}\text { Table. } 4.14 \\
\text { Test of Normality with Chi Square }\left(X^{2}\right)\end{array}$} \\
\hline & & & & & & \multicolumn{2}{|c|}{ Interval } & Class & $\mathbf{Z}_{\text {account }}$ & $\mathbf{Z}_{\text {table }}$ & $\mathbf{L z}_{\text {table }}$ & $\mathbf{E}_{\mathbf{i}}$ & $\mathbf{O}_{\mathrm{i}}$ \\
\hline $17-$ & 7 & 19 & 136 & - & 84,6 & 592, & & Limit & & & & & \\
\hline 22 & 8 & 5 & 5 & 9,2 & 4 & 48 & & 16,5 & $-1,55$ & 0,0606 & & & \\
\hline $23-$ & & 25 , & 204 & - & 10,2 & $\$ 1,9^{7}$ & -22 & & & & 0,1542 & 4,62 & 7 \\
\hline 28 & 9 & 5 & 202 & 3,2 & 4 & 2 & & 22,5 & $-0,79$ & 0,2148 & & & \\
\hline 29 & 3 & 31 & 283 & 28 & 784 & $\begin{array}{r}23 \\
\end{array}$ & -28 & & & & 0,2772 & 8,31 & 8 \\
\hline $29-$ & 2 & $\begin{array}{l}31, \\
5\end{array}$ & 5 & 2,8 & 7,84 & 10,5 & & 28,5 & $-0,02$ & 0,492 & & & \\
\hline 34 & 2 & 5 & 112 & 8,8 & 77,4 & 29 & -34 & & & & 0,2753 & 8,25 & 9 \\
\hline $35-$ & 1 & 37 & 5 & 14 & 4 & 232 & & 34,5 & 0,73 & 0,7673 & & & \\
\hline 40 & & 5 & 87 & 8 & 219. & 3235 & -40 & & & & 0,1659 & 4,97 & 3 \\
\hline $41-$ & & 43 , & 01 & 0 & 04 & 438 & & 40,5 & 1,50 & 0,9332 & & & \\
\hline & & 5 & 49,5 & 20 & son & $08^{41}$ & -46 & & & & 0,0552 & 1,65 & 2 \\
\hline & & & & 8 & 432 & & & 46,5 & 2,27 & 0,9884 & & & \\
\hline $47-$ & & 49 & & & 64 & 43247 & -52 & & & & 0,0104 & 0,31 & 1 \\
\hline 52 & & 5 & & & & 64 & & 52,5 & 3,03 & 0,9988 & & & \\
\hline$\sum$ & $\begin{array}{l}3 \\
0\end{array}$ & & 861 & & & 1848 & & c. & $\begin{array}{l}\text { Accountir } \\
\text { with form }\end{array}$ & 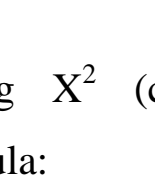 & hi squar & & \\
\hline & & ccou & ting de & eviatic & n stan & lard & & & $\mathrm{X}_{\text {account }}^{2}=$ & $\frac{(O i-E i)^{2}}{E i}$ & & & \\
\hline & & $=\sqrt{\underline{\underline{2}}}$ & $\frac{f(x-\bar{x})^{2}}{\mathrm{~N}}$ & & & & & & $\begin{array}{l}= \\
\frac{(7-4,62)^{2}}{4,62}+\frac{(8-8,31}{8,31}\end{array}$ & $+\frac{(9-8,25)^{2}}{8,25}+\frac{(3-}{2}$ & $\frac{97)^{2}}{97}+\frac{(2-1,65)^{2}}{1,654}$ & $\frac{1-0,31)^{2}}{0,31}$ & \\
\hline & & $=\sqrt{\frac{1}{1}}$ & & & & & & & $\begin{array}{l}=1,22+0 \\
0,07+1,5\end{array}$ & $\begin{array}{l}01+0,06 \\
=3,67\end{array}$ & $+1,78+$ & & \\
\hline & & $=\sqrt{6}$ & & & & & & & & & & & \\
\hline & & & & & & & & & & & & & \\
\hline
\end{tabular}


Volume 2 No. 2, Agustus 2019

d. Looking for degree of freedom (df), with formula:

$$
\begin{aligned}
\text { Df } & =k-3 \\
& =6-3 \\
& =3
\end{aligned}
$$

e. Determining $\mathrm{X}_{\text {table }}^{2}$ with signification $5 \%$ and $\mathrm{df}(3)$

$$
\begin{aligned}
\mathrm{X}_{\text {table }}^{2} & =(1-\alpha)(\mathrm{df}) \\
& =(1-0,05)(3) \\
& =(0,95)(3) \\
& =7.81
\end{aligned}
$$

Based on the calculation above is known that $\mathrm{X}_{\text {account }}^{2}=3,67$ and $\mathrm{X}_{\text {table }}^{2}=$ 7,81 so $X^{2}$ account $=3,67<X_{\text {table }}^{2}=7,81$. The concluded that the test (sample) of the population is normal distribution.

\section{T-Test}

After having getting the data from the post-test score of two

\begin{tabular}{|c|c|c|c|c|c|c|}
\hline & & 7 & 3 & 6 & & \\
\hline 2 & 63 & $\begin{array}{l}1 \\
7\end{array}$ & $\begin{array}{l}- \\
17,6 \\
3\end{array}$ & $\begin{array}{l}- \\
11,9 \\
6\end{array}$ & 310,8 & 143,0 \\
\hline 3 & 67 & $\begin{array}{l}1 \\
7\end{array}$ & $\begin{array}{l}- \\
13,6 \\
3\end{array}$ & $\begin{array}{l}- \\
11,9 \\
6\end{array}$ & 185,7 & 143,0 \\
\hline 4 & 70 & $\begin{array}{l}2 \\
0\end{array}$ & $\begin{array}{l}- \\
10,6 \\
3\end{array}$ & $\begin{array}{l}- \\
8,96\end{array}$ & 112,9 & 80,2 \\
\hline 5 & 70 & $\begin{array}{l}2 \\
0\end{array}$ & $\begin{array}{l}- \\
10,6 \\
3\end{array}$ & $\begin{array}{l}- \\
8,96\end{array}$ & 112,9 & 80,2 \\
\hline 6 & 70 & $\begin{array}{l}2 \\
0\end{array}$ & $\begin{array}{l}- \\
10,6 \\
3\end{array}$ & $\begin{array}{l}- \\
8,96\end{array}$ & 112,9 & 80,2 \\
\hline 7 & 73 & $\begin{array}{l}2 \\
0\end{array}$ & $\begin{array}{l}- \\
7,63\end{array}$ & $\begin{array}{l}- \\
8,96\end{array}$ & 58,2 & 80,2 \\
\hline 8 & 73 & $\begin{array}{l}2 \\
3\end{array}$ & $\begin{array}{l}- \\
7,63\end{array}$ & $\begin{array}{l}- \\
5,96\end{array}$ & 58,2 & 35,5 \\
\hline 9 & 73 & $\begin{array}{l}2 \\
3\end{array}$ & $\begin{array}{l}- \\
7,63\end{array}$ & $\begin{array}{l}- \\
5,96\end{array}$ & 58,2 & 35,5 \\
\hline 10 & 73 & $\begin{array}{l}2 \\
3\end{array}$ & $\begin{array}{l}- \\
7,63\end{array}$ & $\begin{array}{l}- \\
5,96\end{array}$ & 58,2 & 35,5 \\
\hline 11 & 77 & 3 & $\begin{array}{l}- \\
3,63\end{array}$ & $\begin{array}{l}- \\
5,96\end{array}$ & 13,1 & 35,5 \\
\hline 12 & 77 & 2 & - & - & 13,1 & 3,8 \\
\hline
\end{tabular}
classes, then the writer analyzed it by using T-test formula:

Table 4.15

The Calculation Scores of Each Students In Experiment and Control Class

\begin{tabular}{|l|l|l|l|l|l|l|}
\hline $\mathrm{N}$ & $\mathrm{X}_{1}$ & $\mathrm{X}$ & $\mathrm{X}_{1}$ & $\mathrm{X}_{2}$ & $\mathrm{X}_{1}{ }^{2}$ & $\mathrm{X}_{2}{ }^{2}$ \\
$\mathrm{o}$ & & 2 & & & & \\
\hline 1 & 53 & 1 & - & - & 763,4 & 143,0 \\
& & & 27,6 & 11,9 & & \\
\hline
\end{tabular}


Volume 2 No. 2, Agustus 2019

\begin{tabular}{|c|c|c|c|c|c|c|}
\hline & & 7 & 3,63 & 1,96 & & \\
\hline 13 & 77 & $\begin{array}{l}2 \\
7\end{array}$ & $\begin{array}{l}- \\
3,63\end{array}$ & $\begin{array}{l}- \\
1,96\end{array}$ & 13,1 & 3,8 \\
\hline 14 & 80 & $\begin{array}{l}2 \\
7\end{array}$ & $\begin{array}{l}- \\
0,63\end{array}$ & $\begin{array}{l}- \\
1,96\end{array}$ & 0,3 & 3,8 \\
\hline 15 & 80 & $\begin{array}{l}2 \\
7\end{array}$ & $\begin{array}{l}- \\
0,63\end{array}$ & $\begin{array}{l}- \\
1,96\end{array}$ & 0,3 & 3,8 \\
\hline 16 & 80 & $\begin{array}{l}3 \\
0\end{array}$ & $\begin{array}{l}- \\
0,63\end{array}$ & 1,04 & 0,3 & 1,0 \\
\hline 17 & 83 & $\begin{array}{l}3 \\
0\end{array}$ & 2,37 & 1,04 & 5,6 & 1,0 \\
\hline 18 & 83 & $\begin{array}{l}3 \\
0\end{array}$ & 2,37 & 1,04 & 5,6 & 1,0 \\
\hline 19 & 83 & $\begin{array}{l}3 \\
0\end{array}$ & 2,37 & 1,04 & 5,6 & 1,0 \\
\hline 20 & 83 & $\begin{array}{l}3 \\
3\end{array}$ & 2,37 & 4,04 & 5,6 & 16,3 \\
\hline 21 & 87 & $\begin{array}{l}3 \\
3\end{array}$ & 6,37 & 4,04 & 40,5 & 16,3 \\
\hline 22 & 87 & $\begin{array}{l}3 \\
3\end{array}$ & 6,37 & 4,04 & 40,5 & 16,3 \\
\hline 23 & 87 & $\begin{array}{l}3 \\
3\end{array}$ & 6,37 & 4,04 & 40,5 & 16,3 \\
\hline 24 & 90 & 3 & 9,37 & 4,04 & 87,7 & 16,3 \\
\hline 25 & 90 & 3 & 9,37 & 8,04 & 87,7 & 64,6 \\
\hline
\end{tabular}

\begin{tabular}{|l|l|l|l|l|l|l|}
\hline & & 7 & & & & \\
\hline 26 & 90 & 4 & 9,37 & 11,0 & 87,7 & 121,8 \\
& & 0 & & 4 & & \\
\hline 27 & 10 & 4 & 19,3 & 11,0 & 375,1 & 121,8 \\
& 0 & 0 & 7 & 4 & & \\
\hline 28 & 10 & 4 & 19,3 & 14,0 & 375,1 & 197,1 \\
& 0 & 3 & 7 & 4 & & \\
\hline 29 & 10 & 4 & 19,3 & 14,0 & 375,1 & 197,1 \\
& 0 & 3 & 7 & 4 & & \\
\hline 30 & 10 & 5 & 19,3 & 21,0 & 375,1 & 442,6 \\
& 0 & 0 & 7 & 4 & & \\
\hline$\sum$ & & & & & 4117, & 2137, \\
\hline
\end{tabular}

Based on the data above is known that:

$\sum \mathrm{X}_{1}=2419$

$$
\sum \mathrm{X}_{1}^{2}=4117,7
$$

$\sum \mathrm{X}_{2}=869$

$$
\sum \mathrm{X}_{2}^{2}=2137,5
$$

To find the different of using Circle game in teaching English vocabulary between Post-test in experiment class $\left(\mathrm{X}_{1}\right)$ and Post-test in Control class $\left(\mathrm{X}_{2}\right)$ is used the technique t-test as follows:

1. Determine mean of variable $X_{1}$, with formula:

$$
\mathrm{M}_{1}=\frac{\sum \mathrm{X}_{1}}{\mathrm{~N}}
$$




$$
\mathrm{M}_{1}=\frac{2419}{30}=80,63
$$

2. Determine mean of variable $\mathrm{X}_{2}$, with formula:

$$
\begin{aligned}
& \mathrm{M}_{2}=\frac{\sum \mathrm{X}_{2}}{\mathrm{~N}} \\
& \mathrm{M}_{2}=\frac{869}{30}=28,96
\end{aligned}
$$

3. Determine t-test, with formula:

$$
\begin{aligned}
& t_{0}=\frac{\mathrm{M}_{1}-\mathrm{M}_{2}}{\sqrt{\frac{\left(\sum \mathrm{X}_{1}^{2}+\sum \mathrm{X}_{2}^{2}\right)\left(\mathrm{N}_{1}+\mathrm{N}_{2}\right)}{\left(\mathrm{N}_{1}+\mathrm{N}_{2}-2\right)\left(\mathrm{N}_{1} \cdot \mathrm{N}_{2}\right)}}} \\
& t_{0}=\frac{80,63-28,96}{\sqrt{\frac{(4117,7+2137,5)(30+30)}{(30+30-2)(30 \times 30)}}} \\
& t_{0}=\frac{51,67}{\sqrt{\frac{(6255,2)(600)}{(58)(900)}}} \\
& t_{0}=\frac{51,67}{\sqrt{(107,84)(0,06)}} \\
& t_{0}=\frac{51,67}{\sqrt{6,47}} \\
& t_{0}=\frac{51,67}{2,54}=20,34
\end{aligned}
$$

4. Determining $\mathrm{t}$ with signification $5 \%$

$$
\begin{aligned}
\mathrm{df} & =\mathrm{N}_{1}+\mathrm{N}_{2}-2 \\
& =30+30-2 \\
& =58 \\
& =2,00
\end{aligned}
$$

5. Determinug with signification $1 \%$

$$
\begin{aligned}
\mathrm{df} & =\mathrm{N}_{1}+\mathrm{N}_{2}-2 \\
& =30+30-2 \\
& =58 \\
& =2,65
\end{aligned}
$$

From the result of calculation above is known that $\mathrm{t}_{\text {table }}$ with level significance $5 \%=2,00$ and with level significance $1 \%=$ 2,65 , so $\mathrm{t}_{\text {observation }}=20,34>\mathrm{t}_{\text {table }}=2,00$ or $\mathrm{t}_{\text {observation }}=20,34>\mathrm{t}_{\text {table }}=2,65$. The concluded that the writer rejects $H_{0}: t_{0}<t_{t}$ : it means there is no influence of using pronunciation bingo game in teaching English pronunciation and receives $\mathrm{H}_{\mathrm{a}}:>\mathrm{t}_{\mathrm{t}}$ : it means there is influence of using pronunciation bingo game in teaching English pronunciation.

\section{Interpretation of Data}

This analysis purposed to know how far is the influence of using pronunciation to improve students' pronunciation. From the result of research that the mean of pre-test score gained by students in class VII A as experiment class 33,3 was higher than in class VII $\mathrm{B}$ as control class 28,7 . The highest score of pretest in class VIIA as experiment class 53, the lowest score of pre-test in class VIIA as experiment class 20 and the highest score of pre-test in class VIIB as control class 50, the lowest score of pre-test in class VIIB as control class 17 . So, the distribution of scores in class VIIA as experiment class was higher than in class VIIB as control class.

The mean of post-test score gained by students in class VIIA as experiment 
class 80,5 was higher than in class VIIB as control class 28,7. The highest score of post-test in class VIIA as experiment class 100 , the lowest score of post-test in class VIIA class 53 and the highest score of post-test in class VIIB as control score of post-test in class VIIB as control class 17. So, the distribution class VIIA as experiment class was higher than in class VIIB as control class.

From the interpretation of data above can be concluded that using the pronunciation bingo game to improve students' pronunciation will be better and more effective to introduce new pronunciation and to improve students' pronunciation than teaching pronunciation without using pronunciation bingo game.

\section{CONCLUSION}

Based on the research entitled " The Use of Pronunciation Bingo Game To Improve Students' Pronunciation " at The First grade inMTs Alkheriyah Kepandean Ciruas Serang, the writer concludes that :

1. The analysis result about the use of pronunciation bingo game to improve students' pronunciation, has shown that to experiment class is higher that to control class. It means that the use of pronunciation bingo game has significant increase on the improvement of students' pronunciation.

2. From the result of pre-test and post- test between experiment class and control class the writer knows that the students' pronunciation by using pronunciation bingo game is better than students' without using pronunciation bingo game in their pronunciation.

3. On the research analysis, the use of pronunciation bingo game to improve students' pronunciation is applicable for learning pronunciation. It proven by development result of students' pre-test and post-test between experiment class and control class, that shown the application of using pronunciation bingo game.

\section{REFERENCES}

Hadfield ,Jill, Advanced Communication Game, Harlow : DP Press, 1996

Hancock, Mark, Pronunciation Game, Newyork : Cambridge University Press, 1996

Hornby As, Oxford Advanced Learners' Dictionary of Current English, Newyork : Oxford University Press , 1974.

Kelly, Gerald, How to Teach Pronunciation ,England: Lognman , 2000 
Volume 2 No. 2, Agustus 2019

P-ISSN 2623-0062

Universitas Banten Jaya

E-ISSN 2622-9056

Kenworthy ,Joanne , Teaching English

Pronunciation, Newyork :

Logman Group

Nunan David, Methode in Language

Learning, Newyork: Cambridge

University Press, 1992

Rasmusen, Eric ,Games and information, forth Edition,

Australia:Blackwell,2007

Wright, Andrew ,David Betteridge and Michail Bockby,Games For Language Learning Network : Cambridge University Press , 2006 
Volume 2 No. 2, Agustus 2019

P-ISSN 2623-0062

Universitas Banten Jaya

E-ISSN 2622-9056 